\title{
Closing the Gaps: Endoscopic Suturing for Large Submucosal and Full-Thickness Defects
}

\author{
Keshav Kukreja, Suma Chennubhotla, Bharat Bhandari, Ankit Arora and Shashideep Singhal \\ Divisions of Gastroenterology, Hepatology and Nutrition, University of Texas Health Science Center at Houston, Houston, TX, USA
}

This article is a systematic review of relevant literature on endoscopic suturing as a primary closure technique for large submucosal and full-thickness defects after endoscopic mucosal resection (EMR), endoscopic submucosal dissection (ESD), and endoscopic fullthickness resection (EFTR). A comprehensive literature search was conducted through 2016 by using PubMed, to find peer-reviewed original articles. The specific factors considered were the procedural indications and details, success rates, clinical outcomes including complications, and study limitations. Six original articles were included in the final review: two with non-human subjects and four with human subjects. The mean success rate of endoscopic suturing was $97.4 \%$ (100\% for human subjects and $95.4 \%$ for non-human subjects). The procedural time ranged from 7 to $89 \mathrm{~min}$. The average size and depth of lesions were $2.71 \mathrm{~cm}(3.74 \mathrm{~cm}$ [human] and $1.96 \mathrm{~cm}$ [non-human]) and $1.52 \mathrm{~cm}$, respectively. The technique itself had no reported impact on mortality. In conclusion, endoscopic suturing is a minimally invasive technique for the primary closure of defects caused by EMR, ESD, and EFTR, with a high success and low complication rate. Clin Endosc 2018;51:352-356

Key Words: Endoscopic suturing; Endoscopic mucosal resection; Endoscopic submucosal dissection; Submucosal defects; Endoscopic full-thickness defects

\section{INTRODUCTION}

The role of advanced endoscopic resection techniques in the treatment of pre-neoplastic and early neoplastic lesions have been established during the past decade. Endoscopic mucosal resection (EMR) is a well-established minimally invasive therapy for pre-neoplastic gastrointestinal lesions. ${ }^{1}$ Endoscopic submucosal dissection (ESD) and endoscopic full-thickness resection (EFTR) are fast gaining worldwide recognition and becoming more widely used, ${ }^{2-5}$ as they allow

Received: July 19, 2017 Revised: November 24, 2017

Accepted: December 21, 2017

Correspondence: Shashideep Singhal

Division of Gastroenterology, Hepatology and Nutrition, University of Texas Health Science Center at Houston, 6431 Fannin, MSB 4.234, Houston, TX 77030, USA

Tel: +1-713-500-6677, Fax: +1-713-500-6699, E-mail: sdsinghal@gmail.com ORCID: https://orcid.org/0000-0002-8783-4889

(c) This is an Open Access article distributed under the terms of the Creative Commons Attribution Non-Commercial License (http://creativecommons.org/ licenses/by-nc/3.0) which permits unrestricted non-commercial use, distribution, and reproduction in any medium, provided the original work is properly cited. for the acquisition of larger tissue samples, resulting in greater $\mathrm{R} 0$ resection rates and lower instances of local recurrence. ${ }^{6,7}$ Because it involves the intentional creation of a full-thickness defect followed by endoscopic closure, EFTR is the most invasive of the three endoscopic therapies. Therefore, it has an appropriately greater risk of post-procedural adverse events such as bleeding and perforation. ${ }^{8}$ In a review article on ESD, the rate of complications (namely perforation, peritonitis, and bleeding) among gastric, esophageal, and colorectal ESD was noted to be $3.5 \%, 3.3 \%$, and $4.6 \%$, respectively. ${ }^{9}$ In the past, post-procedural complications such as iatrogenic perforation required immediate surgical intervention, resulting in greater costs, increased hospitalization length, and the possibility of additional post-procedural adverse events. However, with the latest advances in endoscopic therapy, the majority of perforations resulting from EMR and ESD can now be managed endoscopically. ${ }^{10}$

One method endoscopists used to achieve closure of EMR and ESD defects was the application of endoscopic clips. However, tissue approximation is not a traditional indication for 
endoscopic clips, as these were created to obtain hemostasis during unintentional intra-procedural bleeding. Employing endoscopic clips for the approximation of larger defects was not only a cumbersome process but also required the application of multiple clips. ${ }^{11}$ As an advancement in defect closure, over-the-scope-clips (OTSCs), which could close defects up to $2 \mathrm{~cm}$, were introduced. ${ }^{12}$ However, OTSCs have two significant limitations: their inability to close larger defects and the difficulty in removing the OTSCs in cases of incomplete resection. In an effort to overcome these deficiencies and decrease the risk of complications, endoscopic full-thickness tissue apposition devices such as endoscopic suturing systems were developed, with the intention of achieving tissue approximation equivalent to that of surgical stitching.

The OverStitch ${ }^{\mathrm{TM}}$ (Apollo Endosurgery Inc., Austin, TX, USA) is one of the commercially available endoscopic suturing devices, ${ }^{13}$ and was created to provide better tissue approximation and control of the depth of suture placement. It incorporates a curved suturing needle ${ }^{14-18}$ that can be reloaded multiple times without requiring scope withdrawal (Fig. 1). ${ }^{14}$ This suturing device has been successfully used in a variety of applications, including closure of persistent gastrocutaneous fistulae, fixation of esophageal stents, suturing of ulcers, and reducing dilated gastrojejunal anastamoses after bariatric surgery. ${ }^{11,14,19-21}$

Studies have demonstrated the feasibility and safety of the immediate closure of large luminal defects by using an endoscopic suturing device with a curved needle. ${ }^{5,22}$ This review includes independent studies, to date, describing the efficacy and utility of endoscopic suturing.

\section{METHODS}

A comprehensive English literature search was completed by using PubMed and Google Scholar to locate peer-reviewed

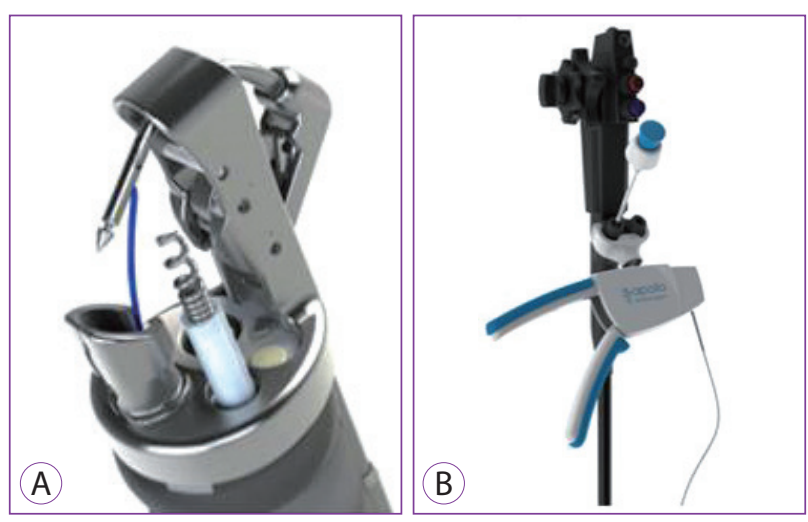

Fig. 1. (A) Overstitch Endcap with Helix. (B) Overstitch device on dual channel endoscope. Used with permission from Apollo Endosurgery, Austin, TX, USA. original and review articles between 2008 and 2016 with the following keywords: endoscopic suturing, submucosal defects, EMR, ESD, and endoscopic full-thickness defects. There were no large case series or randomized control trials available to permit the use of formal systematic review protocols.

Both human and non-human (porcine) studies were included. To further locate additional studies, the references of pertinent studies were also searched individually. The specific factors considered were the procedural indications and details, success rates, clinical outcomes including complications, and study limitations.

\section{RESULTS}

A total of six original peer-reviewed articles were identified. The human and non-human studies included are summarized in Table 1. The studies were performed between 2008 and 2016 in Japan, Italy, Germany, and the United States of America. EMR and EMR were used to treat both benign and malignant lesions including tubular and tubulovillous adenomas, lipomas, and adenocarcinomas of the stomach, rectum, and colon. The average size and depth of lesions were $2.71 \mathrm{~cm}(3.74$ $\mathrm{cm}$ [human] and $1.96 \mathrm{~cm}$ [non-human]) and $1.52 \mathrm{~cm}$, respectively. In total, 17 human subjects and 22 non-human subjects were analyzed in the six studies. There was no reported mortality from the procedure itself.

\section{Technique}

All of the studies employed Overstitch (Apollo Endosurgery, Austin, TX, USA) (Fig. 1A, B), except for one case review by von Renteln et al., in which a Plicator device (NDO Surgical Inc., Mansfield, MA, USA) was used. ${ }^{23}$ All endoscopists were considered technically proficient and used standard Olympus endoscopes. Two studies measured response to suturing in porcine subjects and included a necropsy at the time of follow-up (at 1-2 weeks). ${ }^{16,22}$ EMR, ESD, and EFTR were performed using accepted and standard techniques, with the use of saline containing indigo carmine dye when appropriate, and standard knives (Dual knife, IT-2 knife, and triangle-tip knife; Olympus America Inc., Center Valley, PA, USA). ${ }^{18,22,24,25}$ A unique technique was employed by Rajan et al., in which a submucosal endoscopy with mucosal flap was created to obtain full-thickness gastric biopsies. ${ }^{16}$

In most cases, polypropylene sutures were used owing to their low tissue reactivity and increased durability. The sutures were deployed in a continuous or interrupted manner. The number of sutures deployed ranged from two to four in porcine subjects and from one to two in human subjects. The procedures done on human subjects ranged from 8 to $19 \mathrm{~min}$, 
whereas those carried out on porcine subjects ranged from 7 to $89 \mathrm{~min}$.

\section{Clinical success}

Successful primary closure was defined as complete approximation of the defect with the sutures visualized endoscopically. A visual example of a defect before and after endoscopic suturing is provided in Fig. 2A and B, respectively. A mean success rate of $97.4 \%$ (100\% in human subjects and $95.4 \%$ in non-human subjects) was achieved. No immediate or delayed complications occurred in human subjects at the time of follow-up, which varied from 2 weeks to 5 months among the studies. Minor complications including tarry stools, abscesses, and adhesions were reported in a few porcine subjects (Table 1).

\section{DISCUSSION}

Endoscopic techniques for mucosal, submucosal, and full-thickness resections are becoming increasingly popular as minimally invasive alternatives to surgical interventions. However, for larger, more complex lesions, there is a higher risk of bleeding and perforation. This review summarizes the current evidence for endoscopic suturing as a safe and effective technique for both preventing and managing complications after EMR, ESD, and EFTR. Research on endoscopic suturing with the Overstitch device has shown efficacy in anastomosis dehiscence, fistula closure, stent migration, and iatrogenic perforations after ESD in humans and after full-thickness gastric resection in porcine models. Another utility of endoscopic suturing, as determined in our recent review, is the reduction of dilated gastrojejunal anastomoses, a complication of bariatric surgery. ${ }^{21}$

Our presently reviewed articles include results for both gas- tric and colonic lesions; suturing both before and after endoscopic resection; and resection at mucosal, submucosal, and full-thickness depths. Among the two studies on porcine subjects, greater primary closure rates were observed in the closure of EMR and full-thickness defects than those of ESD., ${ }^{6,16}$ Possibly, the difference between mucosal, submucosal, and full-thickness closure in porcine subjects can be attributed to the increased thickness of the gastric wall and muscularis propria in these animals. Electrocautery in ESD causes scarring and may obstruct the tissue margins, making them less suitable for suturing. Additionally, the use of submucosal injections in ESD to lift the lesion increases the wall thickness and may make suture placement more difficult. Interestingly, von Renteln et al. described two cases in which endoscopic suturing was used to surround the lesion, with the sutures placed underneath, obtaining closure of the gastric wall before snare mucosectomy. ${ }^{23}$ This method firstly prevents the formation of a defect, and also provides an alternative to submucosal injections for lifting a lesion.

Larger studies indicating post-EFTR success with Overstitch in human subjects are lacking; however, the case report by Azzolini et al. suggests its efficacy. ${ }^{24}$ After EFTR, they achieved complete closure with evidence of only a mucosal scar and no complications at the 5-month follow-up. In comparison, the use of Overstitch to correct unintentional full-thickness defects as complications after EMR and ESD is supported by the case study by von Renteln et al. and the retrospective analysis by Kantsevoy et al. ${ }^{23,25}$ Both studies reported complete closure with minimal to no complications. Endoscopic suturing was compared to endoscopic clip placement in five patients, four of whom required laparoscopic colon resection with an ileocolonic anastomosis because of increasing abdominal pain after the procedure. ${ }^{25}$ This indirectly provides support for endoscopic stitching as a viable method for closure in EFTR
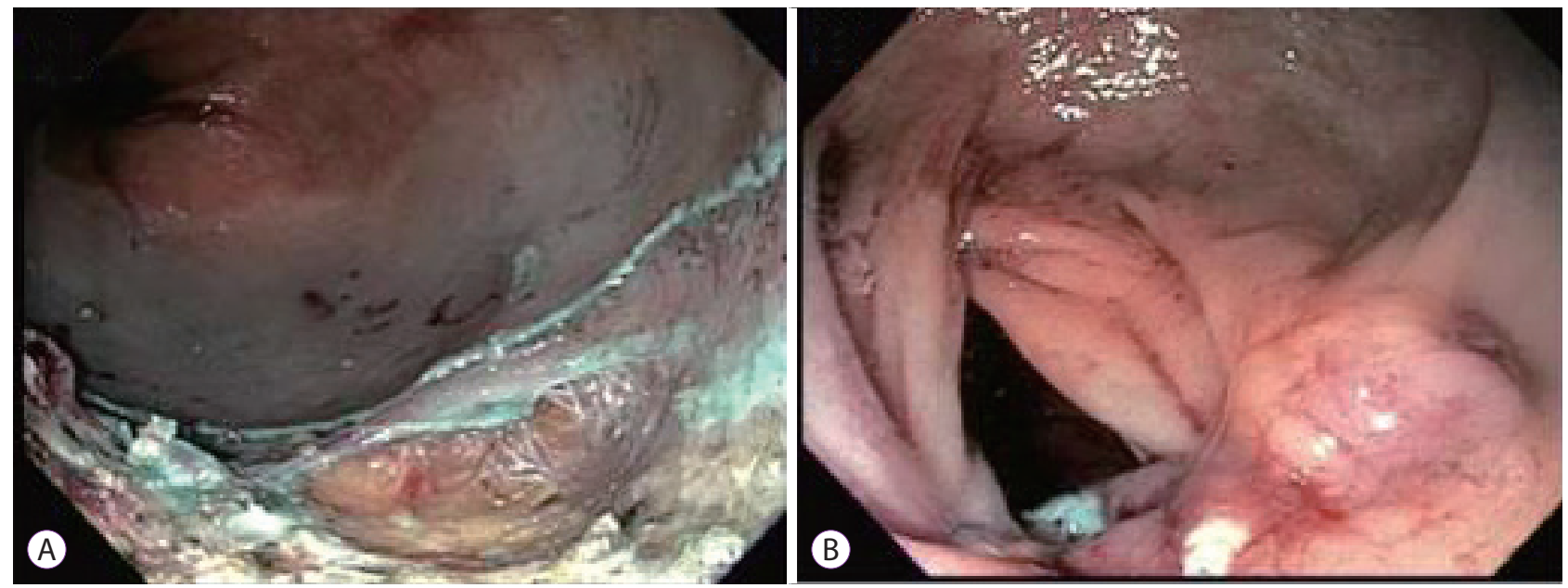

Fig. 2. (A) Large defect following endoscopic submucosal dissection. (B) Following closure of defect with Overstitch device. 


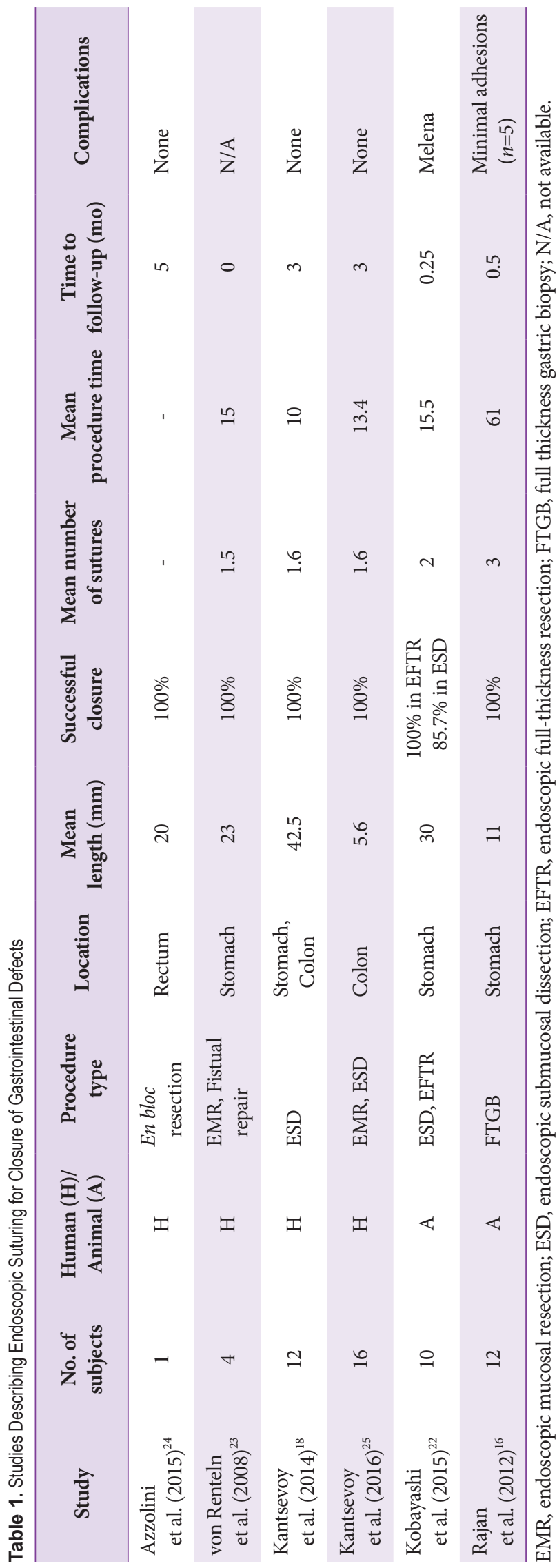

procedures. Furthermore, it emphasizes that conventional hemostatic clips are inappropriate for achieving long-term closure at any level of thickness and should be reserved for obtaining temporary hemostasis.

Although endoscopic suturing has shown utility, it is not without limitations. The currently available endoscopic suturing devices require a double-channel therapeutic endoscope, which is not available in all centers. Furthermore, endoscopic suturing has a considerable learning curve as compared to deploying clips (through-the-scope clips and OTSCs), which all gastroenterologists use routinely. The additional cost of endoscopic suturing devices also needs consideration. With ongoing research, a more defined application of endoscopic suturing can be expected.

\section{CONCLUSIONS}

This review of six original articles supports the notion that endoscopic suturing is a safe and effective method for obtaining closure in both gastric and colonic defects at all levels of thickness. Although more evidence is currently available for endoscopic suturing after EMR and ESD in comparison to EFTR, there is good reason to believe that it would produce similar results. Future studies should focus on assessing endoscopic suturing as a method of achieving closure post-EFTR.

\section{Conflicts of Interest}

The authors have no financial conflicts of interest.

\section{Author Contributions}

Conceptualization: Shashideep Singhal

Data curation: Keshav Kukreja, Suma Chennobhotla, Bharat Bhandari, Ankit Arora

Formal analysis: KK, SC

Investigation: SS

Methodology: SS

Project administration: SS

Resources: KK, SC, BB, AA

Software: KK, SC, BB, AA

Supervision: SS

Validation: SS

Visualization: SS

Writing-original draft: $\mathrm{KK}, \mathrm{SC}, \mathrm{BB}, \mathrm{AA}$

Writing-review\&editing: SS, KK

\section{REFERENCES}

1. Soetikno RM, Gotoda T, Nakanishi Y, Soehendra N. Endoscopic mucosal resection. Gastrointest Endosc 2003;57:567-579.

2. Ono H, Kondo H, Gotoda T, et al. Endoscopic mucosal resection for treatment of early gastric cancer. Gut 2001;48:225-229.

3. Larghi A, Waxman I. State of the art on endoscopic mucosal resection and endoscopic submucosal dissection. Gastrointest Endosc Clin N Am 2007;17:441-469, v. 
4. ASGE Technology Committee, Kantsevoy SV, Adler DG, et al. Endoscopic mucosal resection and endoscopic submucosal dissection. Gastrointest Endosc 2008;68:11-18.

5. Ikeda K, Fritscher-Ravens A, Mosse CA, Mills T, Tajiri H, Swain CP Endoscopic full-thickness resection with sutured closure in a porcine model. Gastrointest Endosc 2005;62:122-129.

6. Oka S, Tanaka S, Kaneko I, et al. Advantage of endoscopic submucosal dissection compared with EMR for early gastric cancer. Gastrointest Endosc 2006;64:877-883.

7. Messmann H, Probst A. Management of endoscopic submucosal dissection complications. Endoscopy 2009;41:712-714.

8. Cao Y, Liao C, Tan A, Gao Y, Mo Z, Gao F. Meta-analysis of endoscopic submucosal dissection versus endoscopic mucosal resection for tumors of the gastrointestinal tract. Endoscopy 2009;41:751-757.

9. Odagiri H, Yasunaga H. Complications following endoscopic submucosal dissection for gastric, esophageal, and colorectal cancer: a review of studies based on nationwide large-scale databases. Ann Transl Med 2017;5:189.

10. Raju GS, Gajula L. Endoclips for GI endoscopy. Gastrointest Endosc 2004;59:267-279.

11. Otake Y, Saito Y, Sakamoto T, et al. New closure technique for large mucosal defects after endoscopic submucosal dissection of colorectal tumors (with video). Gastrointest Endosc 2012;75:663-667.

12. Singhal S, Changela K, Papafragkakis H, Anand S, Krishnaiah M, Duddempudi S. Over the scope clip: technique and expanding clinical applications. J Clin Gastroenterol 2013;47:749-756.

13. Kantsevoy SV, Thuluvath PJ. Successful closure of a chronic refractory gastrocutaneous fistula with a new endoscopic suturing device (with video). Gastrointest Endosc 2012;75:688-690.

14. Kantsevoy SV, Bitner M. Esophageal stent fixation with endoscopic suturing device (with video). Gastrointest Endosc 2012;76:1251-1255.

15. Bonin EA, Wong Kee Song LM, Gostout ZS, Bingener J, Gostout CJ Closure of a persistent esophagopleural fistula assisted by a novel endoscopic suturing system. Endoscopy 2012;44 Suppl 2 UCTN:E8-E9.

16. Rajan E, Gostout CJ, Aimore Bonin E, et al. Endoscopic full-thickness biopsy of the gastric wall with defect closure by using an endoscopic suturing device: survival porcine study. Gastrointest Endosc 2012;76:10141019.

17. Armengol JR, Dot J, Abu-Suboh Abadia M, et al. Full-thickness purely endoscopic resection of colon cancer. Gastrointest Endosc 2012;75(4 Suppl):AB114-AB115

18. Kantsevoy SV, Bitner M, Mitrakov AA, Thuluvath PJ. Endoscopic suturing closure of large mucosal defects after endoscopic submucosal dissection is technically feasible, fast, and eliminates the need for hospitalization (with videos). Gastrointest Endosc 2014;79:503-507.

19. Jirapinyo P, Watson RR, Thompson CC. Use of a novel endoscopic suturing device to treat recalcitrant marginal ulceration (with video). Gastrointest Endosc 2012;76:435-439.

20. Rieder E, Dunst CM, Martinec DV, Cassera MA, Swanstrom LL. Endoscopic suture fixation of gastrointestinal stents: proof of biomechanical principles and early clinical experience. Endoscopy 2012;44:1121-1126.

21. Changela K, Ofori E, Duddempudi S, Anand S, Singhal S. Peroral endoscopic reduction of dilated gastrojejunal anastomosis after bariatric surgery: techniques and efficacy. World J Gastrointest Endosc 2016;8:239243.

22. Kobayashi M, Sumiyama K, Ban Y, et al. Closure of iatrogenic large mucosal and full-thickness defects of the stomach with endoscopic interrupted sutures in in vivo porcine models: are they durable enough? BMC Gastroenterol 2015;15:5.

23. von Renteln D, Schmidt A, Riecken B, Caca K. Gastric full-thickness suturing during EMR and for treatment of gastric-wall defects (with video). Gastrointest Endosc 2008;67:738-744.

24. Azzolini F, Cecinato P, Iori V, De Marco L, Sassatelli R. Endoscopic full-thickness resection for suspected residual rectal neuroendocrine tumor and closure of the defect with a new suturing system. Endoscopy 2015;47 Suppl 1:E556-E557.

25. Kantsevoy SV, Bitner M, Hajiyeva G, et al. Endoscopic management of colonic perforations: clips versus suturing closure (with videos). Gastrointest Endosc 2016;84:487-493. 